\title{
Computer-Based Comparative Analysis of BPSK versus other PSK Modulation Models
}

\author{
B. O. Omijeh ${ }^{1}$, C. E. Adabanya ${ }^{2}$ \\ Department of Electronic \& Computer Engineering, University of Port Harcourt, Choba, Port Harcourt, Nigeria ${ }^{1}$ \\ Centre for Information and Telecommunications Engg, University of Port Harcourt, Choba, Port Harcourt, Nigeria ${ }^{2}$
}

\begin{abstract}
Digital communication has been experiencing a lot of tremendous growth and success. It employs digital technique of modulation and demodulation which makes it more efficient, complex and secure in long distance transmission than its analogue counterpart. Of various types of digital modulation, Binary Phase shift Keying (BPSK) technique of modulation is believed to be reliable in long distance transmission with minimal errors. In this paper, computer based analysis is been carried out to comparatively model and analyse BPSK technique along-side other MPSK techniques over an AWGN channel paying specific attention to the bit error rates(BER), number of errors and number of samples generated by the MATLAB simulation. The MATLAB simulation results show the BPSK method proves to be a better technique where distance and error generation are top factors to be considered.
\end{abstract}

Keywords: Modulation, BPSK, BER, AWGN channel.

\section{INTRODUCTION}

The transit to digital modulation provides more information capacity and unanimity with digital data services, higher data security, better quality communications, and quicker system availability. The aim of a digital communication system is to convey digital data between two or more nodes. Digital modulation techniques possess greater capacity to transport large amounts of traffic than analogue modulation technique[1]

In radio communications this is usually achieved by adjusting a physical characteristic of a sinusoidal carrier, either the frequency, phase, amplitude or a combination thereof by a digital signal usually " 1 "s and "0"s. This is performed in real systems with a modulator at the transmitting end and a detector at the receiving end [2]

The modulator imposes the physical change to the carrier and a demodulator detects the resultant modulation on reception of various Digital modulation techniques [3] BPSK is one of the simplest, most robust of all the PSK techniques .The BPSK modulation and demodulation is designed using MATLAB Simulink [4].
In this paper, implementation of BPSK modulation and demodulation over an AWGN channel has received considerable attention due to their low error rate, high flexibility, low cost, and high speed.

\section{RELATED WORKS}

[5] In their paper, presented why BPSK is normally used for high speed data transfer application, and that it provides a $3 \mathrm{~dB}$ power advantage over the BASK modulation technique and is robust and simple in implementation. It also provides small error rates than any other systems.

[6] in his paper compared the Bit Error Ratio performance when the input of Reed-Solomon (RS) Encoder- Decoder is Integer and Binary to the M-ary frequency shift keying (MFSK) under AWGN, multipath Rayleigh and Rician fading channels. The results show the binary input RS encoder has best BER performance under Rayleigh fading when the value of $\mathrm{M}$ is 2 .

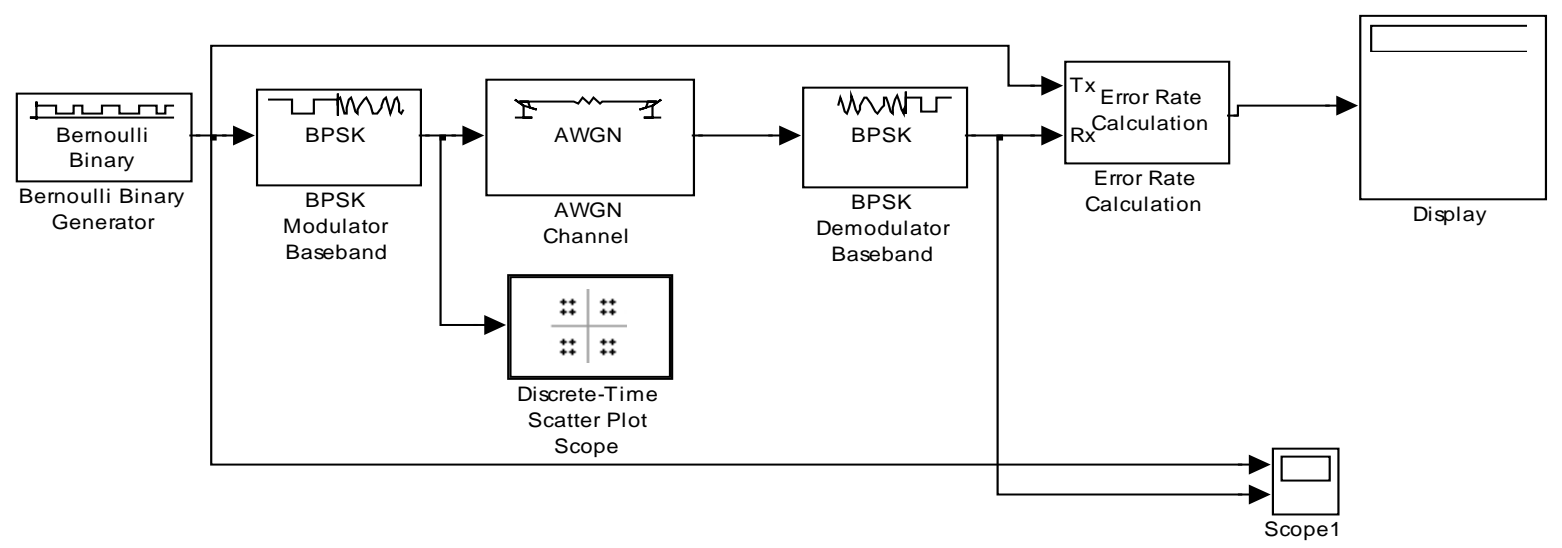

Fig. 1: A TYPICAL BPSK MODEL (Math works, 2009) 
[7] In their paper, the Design of Digital Modulators: - Records were taken for Error rate, Number of Errors BASK, BPSK and BFSK using VHDL, demonstrated the functionality of these digital modulators through generated and Number of Samples for the 2-PSK, 4PSK, 8-PSK and 16-PSK models

simulations using the Quartus II software and experimental measurements of the real-time modulated signal via an oscilloscope [8][9][10].

\section{METHODOLOGY}

- The bpskdoc model in Simulink was enabled and duplicated into three more models replacing the BPSK modulator/demodulator with the 4-PSK, 8-PSK and 16-PSK modulators/demodulators as shown in Fig. 2 below

The following steps were taken to analyse the BPSK - In each model, the option 'stop simulation' was model alongside the other M-PSK models;

- Simulation was run in intervals of 50 Matlab seconds disabled to allow for runtime analyses in the Bit error rate display.
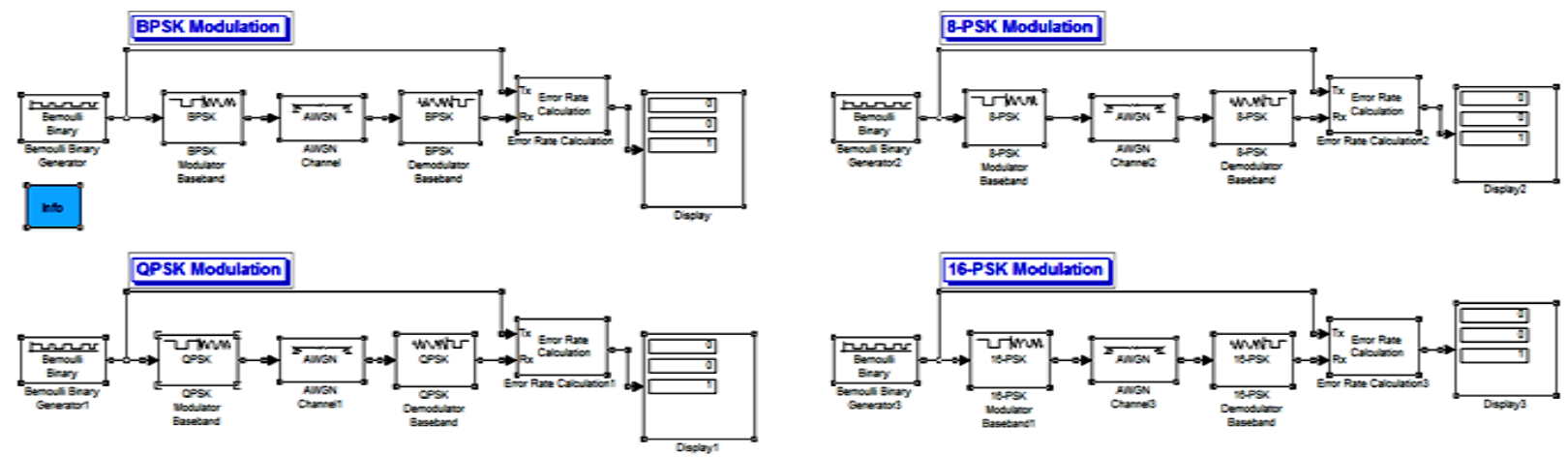

Fig2: Comparative Models for BPSK versus M-PSK

\section{RESULTS AND DISCUSSION}

- $\quad$ The BER (Bit error rate) value for the BPSK modulation Technique was found to be very low and stable over long sample times while the other MPSK techniques had higher and fluctuating values over the same periods. These results are noted in Table .1.

Fig .3 shows the relationship in BER of BPSK in comparison to other MPSK.

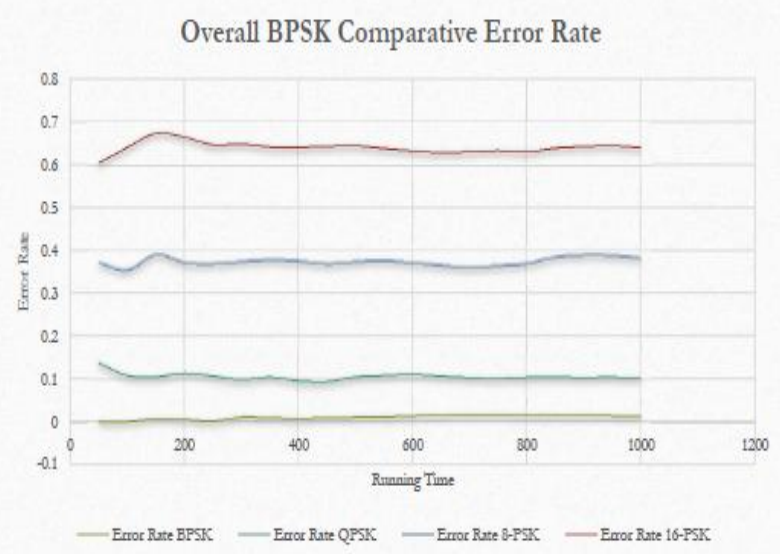

Fig. 3: Overall BPSK Comparative Error Rate

The other MPSK techniques generated errors fasters and in steady growth while the BPSK demonstrated a steady flow generation of errors over long sampling periods.

These results are noted in Table 2 .
TABLE 1: BER results for BPSK, QPSK, 8-PSK and 16-PSK modulation model at increasing simulation time

\begin{tabular}{|c|c|c|c|c|}
\hline \multicolumn{5}{|l|}{ Error Rate } \\
\hline Run Time & BPSK & QPSK & 8-PSK & 16-PSK \\
\hline 50 & 0 & 0.1373 & 0.3725 & $\quad 0.6078$ \\
\hline 100 & 0 & 0.1089 & 0.3564 & 0.6436 \\
\hline 150 & 0.006623 & 0.106 & 0.3907 & 0.6755 \\
\hline 200 & 0.004975 & 0.1144 & 0.3731 & 0.6667 \\
\hline 250 & 0.001195 & 0.1076 & 0.3705 & 0.6494 \\
\hline 300 & 0.009967 & 0.09967 & 0.3754 & 0.6512 \\
\hline 350 & 0.008547 & 0.1054 & 0.3789 & 0.6439 \\
\hline 400 & 0.007481 & 0.09726 & 0.3766 & 0.6434 \\
\hline 450 & 0.00869 & 0.09534 & 0.3703 & 0.6452 \\
\hline 500 & 0.00998 & 0.1058 & 0.3752 & 0.6467 \\
\hline 550 & 0.01089 & 0.1089 & 0.3775 & 0.6407 \\
\hline 600 & 0.01331 & 0.1115 & 0.3727 & 0.6356 \\
\hline 650 & 0.01536 & 0.1075 & 0.3671 & 0.6313 \\
\hline 700 & 0.01569 & 0.1041 & 0.3623 & 0.6334 \\
\hline 750 & 0.01598 & 0.1025 & 0.3662 & 0.6365 \\
\hline 800 & 0.01496 & 0.1049 & 0.3706 & 0.633 \\
\hline 850 & 0.0141 & 0.1058 & 0.3854 & 0.6416 \\
\hline 900 & 0.01443 & 0.1043 & 0.3896 & 0.6448 \\
\hline 950 & 0.01367 & 0.1052 & 0.3891 & 0.6467 \\
\hline 1000 & 0.01299 & 0.1029 & 0.3836 & 0.6424 \\
\hline
\end{tabular}


TABLE 2: No of errors generated from BPSK, QPSK, 8-PSK and 16-PSK modulation model at different simulation time

\begin{tabular}{|c|c|c|c|c|c|}
\hline \multicolumn{6}{|l|}{ No of Errors } \\
\hline Run Time & BPSK & & & & 16-PSK \\
\hline \multicolumn{2}{|c|}{50} & 0 & 7 & 19 & 31 \\
\hline \multicolumn{2}{|c|}{100} & 0 & 11 & 36 & 65 \\
\hline \multicolumn{2}{|c|}{150} & 1 & 16 & 59 & 102 \\
\hline \multicolumn{2}{|c|}{200} & 1 & 23 & 75 & 134 \\
\hline \multicolumn{2}{|c|}{250} & 3 & 27 & 93 & 163 \\
\hline \multicolumn{2}{|c|}{300} & 3 & 30 & 113 & 196 \\
\hline \multicolumn{2}{|c|}{350} & 3 & 37 & 133 & 226 \\
\hline \multicolumn{2}{|c|}{400} & 3 & 39 & 151 & 258 \\
\hline \multicolumn{2}{|c|}{450} & 4 & 43 & 167 & 291 \\
\hline \multicolumn{2}{|c|}{500} & 5 & 53 & 188 & 324 \\
\hline \multicolumn{2}{|c|}{550} & 6 & 60 & 208 & 353 \\
\hline \multicolumn{2}{|c|}{600} & 8 & 67 & 224 & 382 \\
\hline \multicolumn{2}{|c|}{650} & 10 & 70 & 239 & 411 \\
\hline \multicolumn{2}{|c|}{700} & 11 & 73 & 254 & 444 \\
\hline \multicolumn{2}{|c|}{750} & 12 & 77 & 275 & 478 \\
\hline \multicolumn{2}{|c|}{800} & 12 & 84 & 297 & 507 \\
\hline \multicolumn{2}{|l|}{850} & 12 & 90 & 328 & 546 \\
\hline \multicolumn{2}{|c|}{900} & 13 & 94 & 351 & 581 \\
\hline \multicolumn{2}{|c|}{950} & 13 & 100 & 370 & 615 \\
\hline \multicolumn{2}{|l|}{1000} & 13 & 103 & 384 & 643 \\
\hline
\end{tabular}

\section{CONCLUSION}

From the Analysis of BPSK modulation versus the 4-PSK, 8-PSK, and the 16-PSK methods, it was found that the BPSK method demonstrated very low error generation and Bit error rate potential.

Therefore, the BPSK method proves to be a better technique where distance and error generation are top factors to be considered.

\section{REFERENCES}

[1] Haykin, Digital Communication, John Wiley \& Sons, Inc., Replika Press Pvt. Ltd., N. Delhi, India, 2000-2001

[2] Park, J (1978), On binary DPSK detection, IEEE Trans.Communication, [online] Vol26 No 4,(April).pp484 -486.

[3] Sneda ,R \& Pankaj A.(2014),Performance Evaluation of different modulation Techniques on WCDMA For different multipath fading channels.Inter.J.Eng.Res.Tech.Vol1 No2.pp65-69.Available at: www.ijert.com

[4] Shruti, H \& Pawar, M.(2014),BPSK Modulation Technique for Digital Communication.IJAATER.[Online]. pp52-54.

[5] D.K.Sharma ${ }^{1}$, A. Mishra ${ }^{2}$ \& Rajiv Saxena ${ }^{3}$, TECHNIA International Journal of Computing Science and Communication Technologies, VOL. 3, NO. 1, July 2010. (ISSN 0974-3375)

[6] Vaidya (2013),

[7] Neha, P \& Ajay, P. (2013).Design of Digital Modulators. International journal of Advanced Research in computer Science and Software Engineering.[online] Vol 3.(January).p382386. Available from :www.ijarcsse.com.

[8] Sanjui,M \& Tripathi,M.(2013),BPSK and QPSK Modulation Techniques with optimum Tree based interleaver in iterative IDMA Systems.IJCSN [online]Vol 1 No 2..(pp9-15)

[9] Omijeh.B.O and Biebuma.J.J(2013). Traffic Modelling For Capacity Analysis of CDMA Networks using Lognormal Approximation Method, IOSR Journal of Electronics and Communication Engineering (IOSR-JECE), Volume 4, Issue 6 (Jan. - Feb. 2013), PP $42-50$

[10] Omijeh, B.O, R. Okinege, R.Ochi (2013): “Traffic Modelling for capacity analysis of CDMA Networks using Gaussian, International
Journal of Electronic , Communication and Computer Engineering (IJECCE), Vol 5, N0. 3, 2013 